\title{
A Strategy for Quality Control Dissolution Method Development for Immediate-Release Solid Oral Dosage Forms
}

\author{
Gerard M. Bredael ${ }^{1,}{ }^{*}$, Steve Liang ${ }^{2}$, and David Hahn ${ }^{3}$ \\ ${ }^{1}$ Pharmaceutical Sciences and Clinical Supply, Preformulation, Merck, Summit, NJ USA \\ ${ }^{2}$ Analytical Chemistry in Development and Supply, Merck, Summit, NJ USA \\ ${ }^{3}$ Formerly from Pharmaceutical Sciences and Clinical Supply, Analytical Sciences, Merck, Summit, NJ USA
}

e-mail: gerard.bredael@merck.com

\begin{abstract}
Development of an appropriate dissolution method for quality control purposes requires consideration of many factors and a balance of different needs. The method is typically distinctive to each drug product because of the unique characteristics of the active ingredient, the formulation, and the manufacturing process. The method development and selection process remains subjective due to the potential range of profiles that could be considered acceptable. This article presents a strategy and detailed recommendations for developing a quality control dissolution method for immediate-release solid oral dosage forms for the purpose of quality control and formulation or process development. The strategy is developed based on an understanding of dissolution technology, regulatory expectations, literature, and experiences in developing various dissolution methods. A case study demonstrating this strategy with the discriminatory potential of an appropriately developed dissolution method is also described.
\end{abstract}

KEYWORDS: Dissolution; oral drug delivery; formulation; solubility; in vitro models.

\section{INTRODUCTION}

$\mathrm{D}$ issolution testing of solid oral dosage forms has been used for several decades to aid in formulation/ process development and to examine and assure batch-to-batch quality, consistency, and performance of drug products (1). The USP General Chapter <1092> The Dissolution Procedure: Development and Validation has useful explanations on the development aspects. USP General Chapter $<711>$ Dissolution has the specifics for dissolution testing methodology. There are articles that providemore dissolution development background. Skoug et al. (2) gave one of the first comprehensive overviews of the development, validation, and specification setting for dissolution testing. The importance of a compound's biopharmaceutical aspects and a dissolution test design-decision tree based on the Biopharmaceutics Classification System (BCS) was presented by Li et al. (3). Lastly, Gray et al. (4) provided a historical perspective, the broad challenges of development, and applications.

While these resources provide a broad foundation for dissolution method development, this article presents a simple step-by-step strategy for developing a quality control dissolution method for immediate-release solid oral dosage forms. Specific guidance and detailed recommendations are provided. Relevant articles are cited as background for some of the dissolution conditions and development considerations.

*Corresponding author.

\section{MATERIALS AND METHODS Materials}

The compound examined in the case study is a salt form of a weak base. Wet-granulated immediate-release tablets of this compound were used. The dissolution buffers were prepared from analytical grade reagents.

\section{Methods}

The dissolution testing was performed on Distek $2100 \mathrm{C}$ dissolution baths. The paddle method was used with various media. The dissolution analyses were performed in situ with a LEAP Technologies OPT_DISS UV fiber-optic system. The $10-\mathrm{mm}$ probes were used with $320-\mathrm{nm}$ wavelength detection.

\section{DISSOLUTION TEST DEVELOPMENT GUIDE}

A dissolution method development process is described below. Each important parameter of a dissolution test is separated into individual sections to allow easy identification. The strategy itself was created around health authority guidances or guidelines. This guide presents aspects of dissolution method development for ultimately creating a method acceptable to regulatory agencies.

\section{Solubility Based on BCS}

The most important data set for dissolution method development is the solubility-pH profile. The solubility profile will indicate whether the compound is considered a highly soluble compound based on the BCS. If the 
highest proposed strength dosage dissolves in $250 \mathrm{~mL}$ of media over the $\mathrm{pH}$ range 1-6.8 according to the EMA guidance (5) or $\mathrm{pH} 1-7.5$ according to the United States FDA guidance (6), then it is considered a highly soluble compound.

If the compound is highly soluble, dissolution profiles should be established using $900 \mathrm{~mL}$ of $0.1 \mathrm{~N} \mathrm{HCl}, \mathrm{pH} 4.5$, and pH 6.8 media, with typically USP Apparatus 2 (paddles) at $50 \mathrm{rpm}$. The medium that produces the slowest dissolution rate with a standard spindle speed should be selected for the method. A slower dissolution rate will increase the likelihood that the method might be able to discriminate formulation composition, manufacturing process variations, or pharmacokinetics performance. Lastly, the appropriate medium selection would make the method suitable for determining whether the dosage form still would meet BCS Class 3, Class 1, or both criteria for a highly soluble compound over the shelf-life.

If the compound has low solubility across the $\mathrm{pH}$ range, then the initial objective is to develop a dissolution method that dissolves at least $85 \%$ by $30-60 \mathrm{~min}$ (7). Additionally, if an animal PK study has been performed using different API particle sizes, different physical forms, or other critical attributes and PK differences are observed, the method ideally should be capable of rank-ordering the formulations. Subsequent sections provide guidance on how to approach development of an appropriate dissolution test for low-solubility compounds.

\section{Apparatus}

For immediate-release solid oral dosage forms, USP Apparatus 1 (Basket) or Apparatus 2 (paddle) are typically used. The other USP dissolution apparatus are typically used for controlled-release or non-oral formulations. The paddle apparatus should be chosen if the anticipated commercial dosage form will be a non-floating dosage form, unless there are extenuating circumstances. The potential issue with baskets for disintegrating formulations is that the hydrodynamic environment below a basket is not as well mixed as that of the paddle, which may lead to a more challenging interpretation of the dissolution data. Sometimes the dosage form used in an early phase may be different from the final commercial dosage form. If an early dosage form (e.g., capsules) floats, the use of a sinker around the capsule should be considered to allow a paddle method to be used. This will permit the easiest tracking of dissolution data for the conversion from capsule to tablet formulation with the removal of the sinker in the test. It is a good regulatory strategy to minimize dissolution method changes across all phases.
For a floating dosage form, baskets and paddles with sinkers should be evaluated. In some cases, baskets may provide an advantage for nondisintegrating dosage forms. Baskets would create the same hydrodynamic environment reproducibly for these dosage forms as well as ensure that the medium has free access to the dosage form.

\section{Spindle Speed}

With the paddle apparatus, a 50-rpm spindle speed should be used as the starting point based on regulatory guidances from FDA (7), the European Medicines Agency (EMA) (5), and the Japanese Pharmaceutical and Food Safety Bureau (PFSB) (8). If there are issues with coning (the piling of non-dissolving excipients under the paddle that limits media penetration into the pile), the use of paddles with a 75-rpm spindle speed should be investigated. The FDA and PFSB recommend a 75-rpm paddle as an option. The increased paddle speed may disperse excipients better, mimicking in vivo dispersion, and allow unhampered dissolution. A 100-rpm paddle method may be used with sufficient justification, such as eliminating or reducing surfactant concentration.

With the basket apparatus, a spindle speed of $100 \mathrm{rpm}$ should be investigated initially $(5,7,8)$. The $\operatorname{FDA}(7)$ also adds the option of baskets at $50 \mathrm{rpm}$. If dissolution is too rapid to provide a potentially discriminating profile, the 50-rpm basket can be investigated.

Use of spindle speeds other than those recommended in regulatory guidance documents should be considered only when recommended parameters have been exhausted. Use of alternative speeds should be clearly justified. Selecting a different medium (e.g., one that has a higher compound solubility) to accelerate dissolution is preferred to increasing the spindle speed.

A good diagnostic tool for development is an "infinity spin" added to the end of a dissolution method to try to forcibly break apart granules and dissolve any undissolved active pharmaceutical ingredient (API). After the last normal sampling time point, the spindle speed is increased to 150-250 rpm for 15-30 min, and an additional sample is taken. This can provide a quick check on dosage form potency and assure that the dissolution is not solubility limited or that a low dissolution is not due to low potency. However for batch release testing, the added value of an infinity spin is limited.

\section{Media and Buffers}

Dissolution testing with biorelevant media may be useful for internal decision-making purposes during formulation development; however, the QC test could use a completely 
separate test method (9). Biorelevant media are designed to mimic the complexity of human Gl tract solutions and are frequently used during development to better understand a compound's potential in vivo solubility and stability and for formulation screening. Jennifer Dressman at the Johann Wolfgang Goethe University has been involved in researching the complexity of human $\mathrm{Gl}$ solutions and has published multiple articles (10-15) on creating biorelevant solutions with subsequent evaluations using these solutions. Note that methods using biorelevant media are not necessarily biopredictive (linked to a compound's clinical behavior) unless such relationships have been established with clinical study data. QC testing utilizing these biorelevant solutions, however, would be cost-prohibitive, resource demanding, and complex (potential for error). The use of these media increases the difficulty of developing a robust analytical method for QC purposes. For these reasons, simple buffer systems are preferred for routine dissolution analysis. Depending on the compound, such methods may have the potential to be biopredictive.

The type of medium and the volume are selected to provide sink conditions. USP defines sink condition as "the volume of medium at least three times that required in order to form a saturated solution of drug substance." The solubility-versus-pH profile provides the most useful information in medium selection for initial examination. Skoug et al. (2) stated that in vitro in vivo correlations are more likely obtained near the saturated solubility limit. However, as the dissolution method approaches this solubility limit, the risk that the test may be oversensitive also increases while the robustness of the method tends to decrease. Initially selecting a dissolution method with a relatively slow dissolution rate that is still not less than $85 \%$ dissolved by 60 min could provide sufficient discriminatory capability and therefore minimize the risk that a more sensitive test late in the development stage is needed to demonstrate sensitivity to formulation or process changes. Switching to a more discriminatory dissolution method late in development could potentially increase the criticality or encumbrance of such a change.

The general $\mathrm{pH}$ range of dissolution media is from 1.1 to 6.8. The $\mathrm{pH}$ can be higher if needed for solubility reasons. In general, the $\mathrm{pH}$ should not exceed 8.0 (7). A medium is chosen based on the desired $\mathrm{pH}$, for example, hydrochloric acid for $\mathrm{pH}$ 1.0-3.0, glycine for $\mathrm{pH}$ 2.03.0, citrate for $\mathrm{pH} 2.5-3.5$, acetate for $\mathrm{pH} 4.0-5.5$, and phosphate $\mathrm{pH}$ 6.0-8.0. These stated buffer $\mathrm{pH}$ ranges are by no means limitations. A typical dissolution buffer has a 0.05 molar concentration. Unbuffered water is not a preferred medium due to potential variability in $\mathrm{pH}$ depending on the source.
If the medium over the $\mathrm{pH}$ range fails to give adequate dissolution, then surfactants should be evaluated. The first choice for a surfactant is sodium dodecyl sulfate (SDS) that is also called sodium lauryl sulfate (SLS) (7). SDS is the most commonly used surfactant in dissolution media because it is available in high purity with consistency across vendors and is easy to use for accurate concentration preparation. SDS tends to degrade below $\mathrm{pH} 2$ but has successfully been used in such media (16). For these cases, a stability or use period should be provided. A pure form of SDS of at least $98 \%$ purity should be specified. Previously accepted levels of SDS and other surfactants used in a regulatory filing can be found on the FDA dissolution Web site (16).

The concentration of the surfactant used for dissolution test should be justified. Typically the lowest concentration required to achieve an acceptable dissolution profile should be used. An SDS concentration range of 0.1-3\% or higher has been used (16). In unbuffered water, SDS at a concentration below about $0.23 \%$ behaves more as a wetting agent than as a solubilizing agent because it is below its critical micelle concentration. If SDS at a previously approved concentration does not yield at least $85 \%$ dissolved by $60 \mathrm{~min}$ or interacts with the active/ excipients, other surfactants listed on this FDA Web site (16) should be examined.

The effect of medium deaeration should be investigated. Room-temperature medium can hold more dissolved gases than medium at $37^{\circ} \mathrm{C}$. When the medium is heated, the dissolved gases have a tendency to form bubbles. These bubbles could have an unpredictable effect on dissolution. The bubbles may cause a dosage form to adhere to the apparatus/vessel, reduce medium access to particles, or increase the tendency for particles to float. A significant effect could be observed in variability or a change in the dissolution rate.

\section{Medium Volume}

The standard dissolution medium volumes used in the industry and accepted by regulatory agencies are $500 \mathrm{~mL}$ and $900 \mathrm{~mL}$. These volumes are selected to provide sink conditions for the compound and do not represent the volumes of liquid encountered by the product in vivo. Indepth reviews of the human gastrointestinal physiology are documented by McConnell and Mudie $(17,18)$.

Smaller dissolution medium volumes can be used during development to reduce API supply requirements, and larger volumes up to $4 \mathrm{~L}$ may be used if required for sink-solubility reasons. In either case, an appropriate apparatus is required. The dissolution behavior (variability and profile) of the dosage form itself will be the best 
guide in choosing the volume. An effort to use one of the two standard volumes should be made to facilitate method transfer and reduce the likelihood of regulatory questions.

It is preferred to have one method and therefore one volume across all strengths of a compound. This allows the evaluation of the profiles across strengths and a check on the dissolution of same/similar formulations. Such a method shows whether the higher and lower strengths are dissolving at similar rates in vitro. Additionally, use of the same method across dosage strengths may provide opportunities for bracket-testing of only the high and low strengths under certain circumstances.

\section{Sampling Time Points}

During development of IR products, sampling time points generally range from $5 \mathrm{~min}$ to more than $60 \mathrm{~min}$. The 5-min time point may be used for suspensions or other fast dissolving formulations where the variation is not high. Typical time points are 15, 30, 45, and $60 \mathrm{~min}$. However, the time points are based on the product's profile and on the method's ability of tracking key aspects of the formulation. If there is a desire to better understand or track the disintegration effect, a 5- or 10-min time point would be needed. (For fast-dissolving products, a fiberoptic dissolution system could test more time points to obtain a better defined dissolution profile.) If there is a significant risk of slowing on stability, a time point may be added beyond $60 \mathrm{~min}$ to ensure that at least $85 \%$ dissolved average values are achieved.

It is useful to have a sampling time point at $15 \mathrm{~min}$, especially if the compound is dissolved at least $85 \%$ within that period of time. If this is achieved in $0.1 \mathrm{~N} \mathrm{HCl}$, the FDA (7) considers that the dosage "behaves like a solution and generally should not have any bioavailability problems.". It is also the specified time point and specification for a potential BCS 3 biowaiver (5). Finally, in comparing dissolution profiles, an $f_{2}$ calculation, which is a logarithmic transformation of the sum-squared error differences between the dissolution curves, is not required for similarity justification with at least $85 \%$ dissolved (6) or greater than $85 \%$ dissolved (5) in $15 \mathrm{~min}$.

\section{Sinkers}

As mentioned previously, sinkers can be utilized on capsules to allow use of the USP 2 Apparatus. Sinkers may also aid in other situations, such as sticky tablets or slowly disintegrating tablets. Tablets sticking to vessels may result in high variability in dissolution profiles because they may stick at various off-center locations in the vessels. The non-centered tablets are exposed to a different hydrodynamic environment than those that are centered $(19,20)$. Slowly disintegrating tablets may need fluid flowing around the tablets to generate more consistent dissolution profiles. Placing the dosage forms in sinkers may resolve these concerns, allowing the use of the paddle apparatus. Various sinker configurations and models should be tested to find one that gives the desired results. Lastly, the sinker configuration or model should be specified in the method due to the potential effects that different sinker models may have on the hydrodynamics surrounding the dosage form.

\section{Filtration and Endpoint Analysis}

Filtration of dissolution samples should eliminate post-sampling dissolution of API particles and reduce the potential that excipient particles might create backpressure/clogging issues in the analytical instrumentation. Typical filter pore sizes range from 0.45 to $70 \mu \mathrm{m}$. For micronized drug substance, the analyst should strive to utilize the filter with the smallest feasible pore size .

The analytical method will depend on the dosage form, amount of compound, and compound UV absorptivity. UV analysis using a fiber-optic or online instrument is the most efficient technique available for dissolution sample analysis, when applicable. Also, online UV or fiber-optic analysis will provide the dissolution results at the end of the dissolution test for fast turnaround time. Applicability of UV methods depends on both suitable absorptivity and absence of significant capsule shell or excipient interference. HPLC/UPLC analysis is the other typical method. With regard to HPLC, it is better to inject larger volumes to maintain sensitivity for lower strength dosages than to decrease the dissolution medium volume to increase HPLC response.

\section{Robustness}

The effect of the various dissolution parameters $(\mathrm{pH}$, surfactant concentration, etc.) on the dissolution profiles should be mapped to assess robustness during development. For example, the dissolution robustness with regard to minor changes in the $\mathrm{pH}$ should be checked. A deviation of $\pm 0.05 \mathrm{pH}$ units should have no significant impact on the dissolution rate. If there is, a different $\mathrm{pH}$ should be chosen for robustness reasons.

\section{Evaluation of Method Sensitivity/Discriminatory Power}

Batches prepared from variants of the target commercial formulation levels or processing parameters are typically used to analyze the sensitivity or discriminatory power of the dissolution method. Among the factors for consideration are drug substance particle size, formulation composition (such as low disintegrant), and a process variation (such as over-granulation). The variants 
would be characterized using the proposed dissolution method, and adequate in vitro discrimination should be demonstrated. What is considered adequate sensitivity will vary from project to project.

\section{Subsequent Development}

Table 1 is a summary of the key recommendations for developing an early phase dissolution method. Development of a dissolution method progresses as the method is continually optimized based on the results of its sensitivity to critical formulation/process characterization changes or any clinical PK study with varied formulations. During development, formulation modifications or other formulations may be tested in the clinic. The clinical PK results should always be examined with regard to the potential of optimizing the dissolution test for in vitro-in vivo correlation (IVIVC). A dissolution test is optimized by modifying the test to better replicate the in vivo outcome.

Table 1. Dissolution Test Parameters and Recommendations

\begin{tabular}{|l|l|}
\hline Parameter & Recommendation \\
\hline Volume & $\begin{array}{l}900 \mathrm{~mL} \text { or } 500 \mathrm{~mL} \\
\text { Other volume with justification }\end{array}$ \\
\hline Medium & $\begin{array}{l}\text { Typically } \geq 3 \times \text { saturation level } \\
\text { SDS as first surfactant of choice } \\
\text { FDA Web site for other surfactants and levels }\end{array}$ \\
\hline Apparatus & $\begin{array}{l}\text { Paddles: } 50 \text { rpm or } 75 \mathrm{rpm} \text { if coning occurs } \\
\text { Baskets: } 100 \text { rpm or } 50 \text { rpm for rapid dissolution } \\
\geq 85 \% \text { dissolved by } 30-60 \text { min }\end{array}$ \\
\hline
\end{tabular}

Note that when two dissimilar formulations are used in a study, it should not be expected that a dissolution test would necessarily mimic the in vivo trend. This is because the critical formulation variables may be different for each formulation, and a universal IVIVC that applies to both formulations should not be expected. If modifications of a specific formulation type are tested in the clinic, then these results can be used to potentially direct the dissolution test modifications to create an IVIVC. This IVIVC development strategy, the determination if an IVIVC study is warranted, and batch selection process are detailed in a recent publication (21).

\section{CASE STUDY}

A dissolution method development case study (without in vivo data) on an IR wet-granulated tablet dosage is presented. The compound is the salt form of a weak base and has the $\mathrm{pH}$ solubility profile as displayed in Figure 1. It is soluble only under acidic conditions. As is typical for a standard IR formulation, the API particle size can be a critical quality attribute for the formulation. The medium selection was optimized with regard to the dissolution of the tablets formulated with two different API particle sizes as displayed in Figure 2. The standard 50-rpm paddle speed with the standard $900-\mathrm{mL}$ volume was used with varied $\mathrm{pH}$ media. The $\mathrm{pH} 3.0$ medium provided sink conditions and was the most discriminating with regard to API particle size.

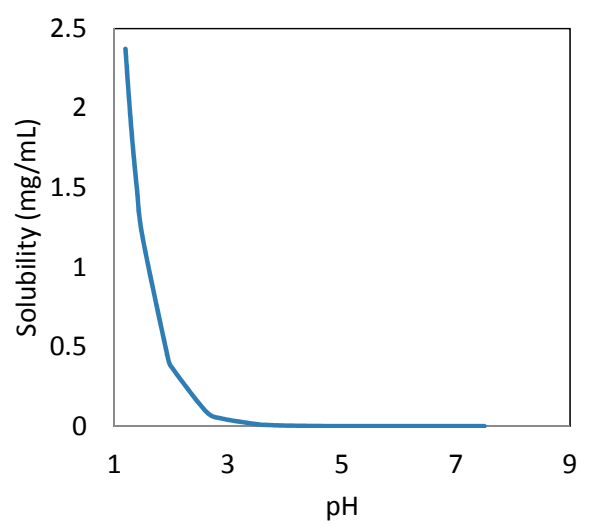

Figure 1. Solubility profile of the salt form of the compound vs solution $\mathrm{pH}$.

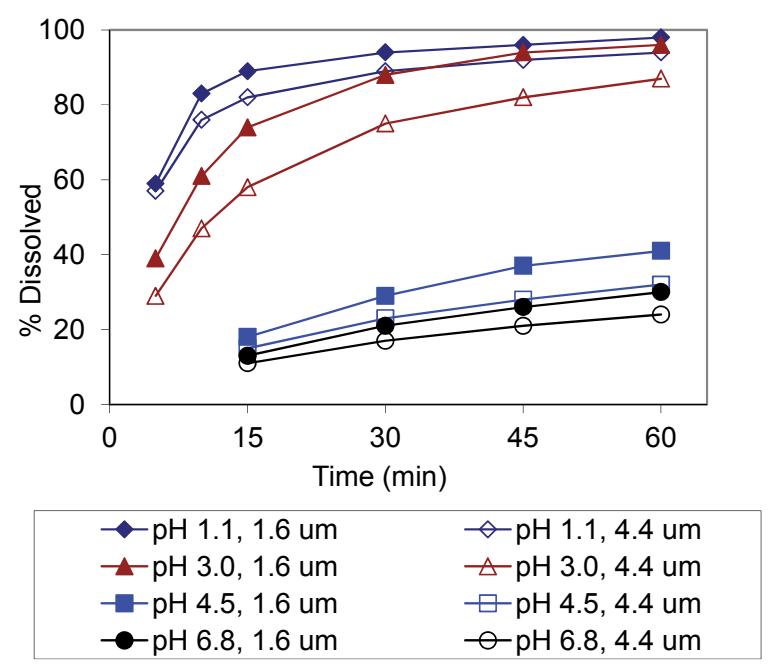

Figure 2. Dissolution profiles showing the effect of medium $\mathrm{pH}$ on tablets with two different API PS, $D_{50}=1.6$ and $4.4 \mu \mathrm{m}$ (900 mL with $50 \mathrm{rpm}$ paddles).

The second critical quality attribute to be examined was the compound's physical state. The compound is in salt form, but upon exposure to humidity, can potentially be converted to the free base form. The discriminatory ability of various $\mathrm{pH}$ media was investigated with two tablet batches with different percentages of salt to free base conversion as displayed in Figure 3. The pH 3.0 
medium was the most discriminating while still satisfying the development criterion of at least $85 \%$ dissolved by 60 min. The pH 3.5 medium does not dissolve at least $85 \%$ within that period of time. The dissolution profiles in $\mathrm{pH}$ 3.0 media for tablets with a wide range of percentage form change are shown in Figure 4. The dissolution rate decreased with an increase in percentage form change. Various batches were examined with this dissolution

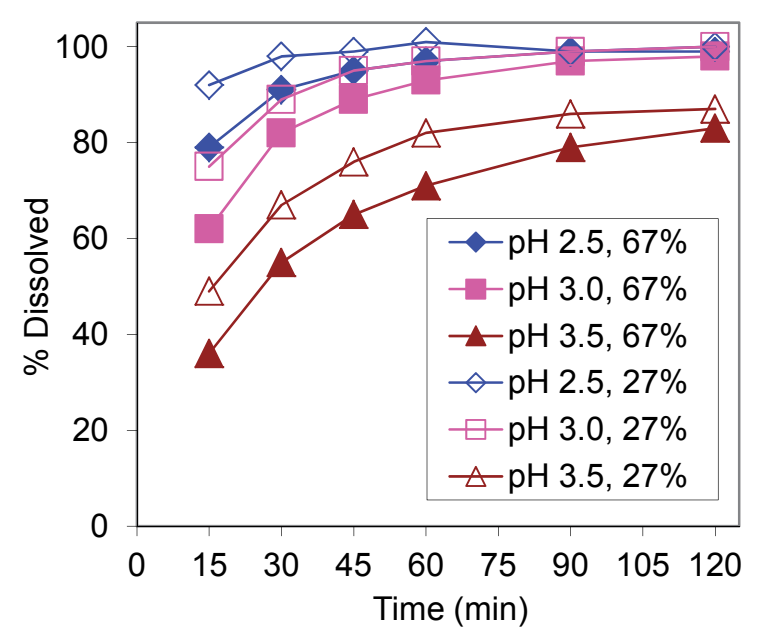

Figure 3. Dissolution profiles showing the effect of medium $\mathrm{pH}$ on tablets with two different percentages of API form change, $27 \%$ and $67 \%$ (900 $\mathrm{mL}$ with $50 \mathrm{rpm}$ paddles).

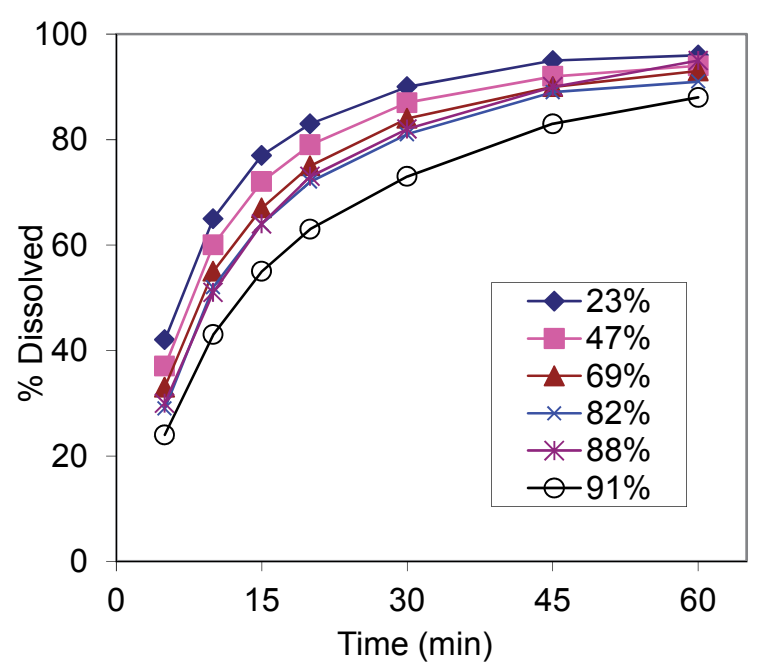

Figure 4. Dissolution profiles from tablets with varying API form change percents. (Dissolution method: $50 \mathrm{rpm}$ paddles with $900 \mathrm{~mL}$ of $\mathrm{pH} 3.0$ medium.)

method, and the dissolution averages are plotted against form change percentages in Figure 5. A linear relationship is achieved over the range of form-change levels. In summary, the selected dissolution method utilizing $50 \mathrm{rpm}$ paddles with $900 \mathrm{~mL}$ of $\mathrm{pH} 3.0$ media has demonstrated sensitivity or discrimination to the two main critical attributes of the formulation and is justified.

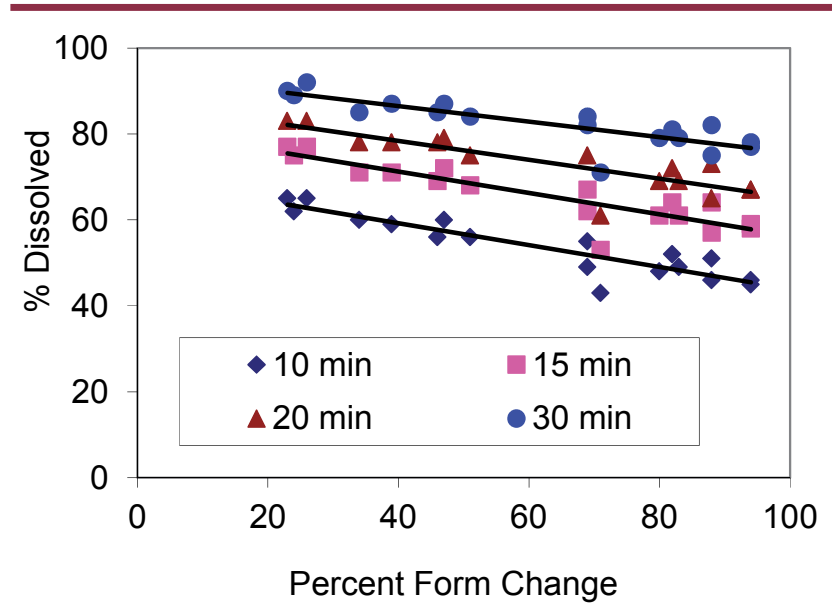

Figure 5. Relationship between percent dissolved and percent API form change. (Dissolution method: $50 \mathrm{rpm}$ paddles with $900 \mathrm{~mL}$ of $\mathrm{pH} 3.0$ medium.)

\section{CONCLUSIONS}

This article outlines a strategy and detailed recommendations for the development of a quality control dissolution test. The development of a dissolution test is dependent on the characteristics of the compound and its formulation. The described strategy is built upon the framework supplied by health authorities from around the world. An IR dissolution method development case study indicating the potential discriminatory potential of a dissolution method to critical quality attributes was provided. A dissolution test method should be optimized based upon sensitivity to critical formulation/process variables and linked, when possible, to actual in vivo responses.

\section{ACKNOWLEDGMENTS}

The authors wish to thank the following scientists who had input in preparing this article: Lee Dowden, Moniek van der Eem, Francis Flanagan, and Hu (Jerry) Wang.

\section{CONFLICT OF INTEREST}

No conflict of interest has been declared by the authors.

\section{REFERENCES}

1. Dokoumetzidis, A.; Macheras, P. A century of dissolution research: From Noyes and Whitney to the Biopharmaceutics Classification System. Int. J. Pharm. 2006, 321 (1-2), 1-11. DOI: 10.1016/j. ijpharm.2006.07.011.

2. Skoug, J. W.; Halstead, G. W.; Theis, D. L.; Freeman, J. E.; Fagan, D. T.; Rohrs, B. R. Strategy for the 
development and validation of dissolution tests for solid oral dosage forms. Pharm. Tech. 1996, 20 (5), 58-72.

3. Li, S.; He, H.; Parthiban, L. J.; Yin, H.; Serajuddin, T. M. IV-IVC Considerations in the Development of Immediate-Release Oral Dosage Form. J. Pharm. Sci. 2005, 94 (7), 1396-1417. DOI: 10.1002/jps.20378.

4. Gray, V.; Kelly, G.; Xia, M.; Butler, C.; Thomas, S.; Mayock, S. The Science of USP 1 and 2 Dissolution: Present Challenges and Future Relevance. Pharm. Res. 2009, 26 (6), 1289-1302. DOI: 10.1007/s11095008-9822-x.

5. Guideline on the Investigation of Bioequivalence; CPMP/EWP/QWP/1401/98 Rev. 1; Committee for Medicinal Products for Human Use (CHMP), European Medicines Agency: London, 2010.

6. Waiver of In Vivo Bioavailability and Bioequivalence Studies for Immediate-Release Solid Oral Dosage Forms Based on a Biopharmaceutics Classification System; Guidance for Industry; U.S. Department of Health and Human Services, Food and Drug Administration, Center for Drug Evaluation and Research (CDER), U.S. Government Printing Office: Washington, DC, 2000.

7. Dissolution Testing of Immediate Release Solid Oral Dosage Forms; Guidance for Industry; U.S. Department of Health and Human Services, Food and Drug Administration, Center for Drug Evaluation and Research (CDER), U.S. Government Printing Office: Washington, DC, 1997.

8. Guideline for Bioequivalence Studies of Generic Products; English translation of Attachment 1 of Division-Notification 0229 No. 10; Pharmaceutical and Food Safety Bureau, Ministry of Health, Labour and Welfare, Government of Japan: Tokyo, 2012.

9. Azarmi, S.; Roa, W.; Löbengerg, R. Current perspectives in dissolution testing of conventional and novel dosage forms. Int. J. Pharm. 2007, 328 (1), 12-21. DOI: 10.1016/j.ijpharm.2006.10.001.

10. Jantratid, E.; Janssen, N.; Reppas, C.; Dressman, J. B. Dissolution Media Simulating Conditions in the Proximal Human Gastrointestinal Tract: An Update. Pharm. Res. 2008, 25 (7), 1663-1676. DOI: 10.1007/ s11095-008-9569-4.

11. Kalantzi, L.; Goumas, K.; Kalioras, V.; Abrahamsson, B.; Dressman, J. B.; Reppas, C. Characterization of the Human Upper Gastrointestinal Contents Under Conditions Simulating Bioavailability/Bioequivalence Studies. Pharm. Res. 2006, 23 (1), 165-176. DOI: 10.1007/s11095-005-8476-1.

12. Vertzoni, M.; Dressman, J.; Butler, J.; Hempenstall, J.; Reppas, $C$. Simulation of fasting gastric conditions and its importance for the in vivo dissolution of lipophilic compounds. Eur. J. Pharm. Biopharm. 2005, 60 (3), 413-417. DOI: 10.1016/j.ejpb.2005.03.002.

13. Klein, S.; Dressman, J. B.; Butler, J.; Hempenstall, J. M.; Reppas, C. Media to simulate the postprandial stomach I. Matching the physicochemical characteristics of standard breakfasts. J. Pharm. Pharmacol. 2004, 56 (5), 605-610. DOI: 10.1211/0022357023367.

14. Dressman, J. B.; Amidon, G. L.; Reppas, C.; Shah, V. P. Dissolution Testing as a Prognostic Tool for Oral Drug Absorption: Immediate Release Dosage Forms. Pharm. Res. 1998, 15 (1), 11-22. DOI: 10.1023/A:1011984216775.

15. Mithani, S. D.; Bakatselou, V.; TenHoor, C. N.; Dressman, J. B. Estimation of the Increase in Solubility of Drugs as a Function of Bile Salt Concentration. Pharm. Res. 1996, 13 (1), 163-167. DOI: 10.1023/A:1016062224568.

16. Dissolution Methods Database. U.S. Food and Drug Administration Web site. http://www.accessdata. fda.gov/scripts/cder/dissolution/index.cfm (accessed June 19, 2015).

17. McConnell, E. L.; Fadda, H. M.; Basit, A. W. Gut instincts: Exploration in intestinal physiology and drug delivery. Int. J. Pharm. 2008, 364 (2), 213-226. DOI: 10.1016/j.ijpharm.2008.05.012.

18. Mudie, D. M.; Amidon, G. L.; Amidon, G. E. Physiological Parameters for Oral Delivery and in Vitro Testing. Mol. Pharmaceutics 2010, 7 (5), 1388-1405. DOI: $10.1021 / \mathrm{mp} 100149 \mathrm{j}$.

19. Wang, B.; Bredael, G.; Armenante, P. M. Effect of a Fiber-Optic Probe on the Dissolution of Salicylic Acid Tablets in USP Apparatus 2. Dissolution Technol. 2013, 20 (2), 21-30. DOI: 10.14227/DT200213P21.

20. Zhang, Y.; Bredael, G.; Armenante, P. Dissolution of Prednisone Tablets in the Presence of an ArchShaped Fiber Optic Probe in a USP Dissolution Testing Apparatus 2. J. Pharm. Sci. 2013, 102 (8), 2718-2729. DOI: 10.1002/jps.23651.

21. Bredael, G. M.; Bowers, N.; Boulineau, F.; Hahn, D. In Vitro-In Vivo Correlation Strategy Applied to an Immediate-Release Solid Oral Dosage Form with a Biopharmaceutical Classification System IV Compound Case Study. J. Pharm. Sci. 2014, 103 (7), 2125-2130. DOI:10.1002/jps.24036. 